\title{
Magnetic Fields in Galaxies
}

\author{
Ellen G. Zweibel ${ }^{1}$ \\ ${ }^{1}$ Departments of Astronomy \& Physics, \& Center for Magnetic Self-Organization \\ University of Wisconsin-Madison \\ 475 N. Charter St, Madison, Wisconsin 53706 USA \\ email: zweibel@astro.wisc.edu
}

\begin{abstract}
The origin and evolution of magnetic fields in the Universe is a cosmological problem. Although exotic mechanisms for magneotgenesis cannot be ruled out, galactic magnetic fields could have been seeded by magnetic fields from stars and accretion disks, and must be continuously regenerated due to the ongoing replacement of the interstellar medium. Unlike stellar dynamos, galactic dynamos operate in a multicomponent gas at low collisionality and high magnetic Prandtl number. Their background turbulence is highly compressible, the plasma $\beta \sim 1$, and there has been time for only a few large exponentiation times at large scale over cosmic time. Points of similarity include the importance of magnetic buoyancy, the large range of turbulent scales and tiny microscopic scales, and the coupling between the magnetic field and certain properties of the flow. Understanding the origin and maintenance of the large scale galactic magnetic field is the most challenging aspect of the problem.
\end{abstract}

Keywords. galaxies:magnetic fields, cosmology:miscellaneous,plasmas

\section{Introduction}

Most of the papers presented at this symposium are about the Sun and other stars, the topics on which Juri Toomre has concentrated for most of his career. This paper is about how magnetic fields in galaxies originated, and how they evolve. Because many aspects of this topic have recently been reviewed elsewhere in great detail (Widrow 2002, Kulsrud \& Zweibel 2008, Beck 2009, Han 2009), I will focus on contrasting the stellar and galactic dynamo problems. I will write from a personal perspective, and will not attempt to give a comprehensive list of references. The paper is organized as follows: $\S 2$ briefly reviews existing theories of and observational constraints on cosmic magnetogenesis, $\S 3$ does the same for galactic magnetic fields, $\S 4$ discusses similarities and differences between the stellar and galactic dynamo problems, $\S 5$ discusses the likely ingredients of galactic dynamo theory, and $\S 6$ is a summary.

\section{Cosmic Magnetogenesis}

The origin of magnetic fields is a cosmological problem. It is widely accepted that magnetic fields were not produced in the Big Bang, but later. It is useful to classify theories of magnetogenesis as top-down and bottom-up. In top-down theories, the entire Universe is magnetized by a global process. Some of these theories are based on new and as yet unconfirmed physics. Others rely on conventional plasma processes, such as the Biermann Battery. Examples of top down theories based on conventional physics are magnetization by the Biermann battery in cosmological ionization fronts or cosmological shock fronts (Kulsrud et al. 1997, Gnedin et al. 2000). In bottom up theories, magnetic fields are generated in small objects and propagated to large scales by a combination of outflows, free expansion, and diffusion. Examples are magnetization of galaxies by 
magnetized stellar ejecta (Rees 1987), and magnetization of portions of the intergalactic medium by fossil radio lobes from AGN (Fulanetto \& Loeb 2001, Kronberg et al. 2001).

The best evidence for a top down theory would be the discovery of a pervasive intergalactic magnetic field. For many years there were only rather high upper limits on the existence of such a field. One is an upper limit on the magnetic pressure at the time of Big Bang Nucleosynthesis; this corresponds to a field at the current epoch of about $10^{-7} \mathrm{G}$, which is about $1 \%-10 \%$ of the fieldstrengths measured in galaxies. Limits on a coherent field come from the lack of evidence for an increase in Faraday rotation with redshift in some particular direction, and are 2-3 orders of magnitude smaller.

Recent high resolution observations of extragalactic, pointlike $\mathrm{TeV} \gamma$-ray sources has made it feasible to search for much weaker magnetic fields. If the intergalactic medium is weakly magnetized, then $e^{ \pm}$pairs created by collisions between $\mathrm{TeV}$ and ambient photons will gyrate through a small angle before producing additional $\gamma$ photons by inverse Compton scattering. This produces a $\gamma$-ray helo around the original source.

Recently Neronov \& Vovk (2010) have set a lower limit of $310^{-16} \mathrm{G}$ for large scale fields along the line of sight to two TeV blazar sources observed by the Fermi satellite, while Ando \& Kusenko (2010) claim to have detected a field of $10^{-15} \mathrm{G}$, also from Fermi. As weak as these fields are, they exceed the minimum predictions for fields produced by the Biermann Battery, as we shall now see.

According to a simple variant of the battery mechanism, an electron pressure gradient which arises, e.g. due to ionizing radiation, must be balanced by an electric field

$$
e n_{e} E=-\nabla P_{e} \text {. }
$$

From Faraday's Law, the rate of change in the magnetic flux $\Phi$ threading a surface moving with the fluid is then

$$
\frac{d \Phi}{d t}=\frac{c}{e} \int_{C} \frac{\nabla P_{e} \cdot d l}{n_{e}},
$$

where $C$ is the contour bounding the surface. If the contours of constant $n_{e}$ are also contours of constant $P_{e}$, then the integrand in eqn. (2.2) can be written as an exact differential, and vanishes. But if $n_{e}$ and $P_{e}$ are not functions of one another - which for an ideal gas implies the that gradients of $n_{e}$ and $T_{e}$ are nonparallel - there is a net EMF around $C$, which allows creation of magnetic flux.

It can also be estimated from Faraday's Law that the ion gyrofrequency $\omega_{c i} \equiv Z e B / m_{i} c$ evolves according to

$$
\omega_{c i} \sim \frac{v_{i}^{2} t}{l_{n} l_{T}},
$$

where $v_{i}$ is the ion thermal velocity, $t$ is time, and $l_{n}$ and $l_{T}$ are the electron density and temperature lengthscales, respectively. In a disk geometry, where one of the $l$ is of order the radius $R$ and the other of order the scale height $H$, we estimate that the ratio of gyrofrequency to rotation frequency, $\omega_{c i} / \Omega$, satisfies $\omega_{c i} / \Omega \sim \Omega t(H / R)$. That is, $R / H$ rotations must elapse before the ions are magetized. This argument, and the inverse dependence of $\omega_{c i}$ on $l_{n}$ and $l_{T}$, makes it clear that the Biermann battery can make appreciable magnetic fields only in small objects, likes stars or accretion disks. Numerical calculations of the battery on cosmological scales find fieldstrengths on the order of $10^{-18}-10^{-21} \mathrm{G}$. It should be mentioned that the simulations in question have rather coarse spatial resolution. Small scale structure produces stronger fields, in accord with eqn. (2.3). But it is interesting that the large scale intergalactic fields probed by $\gamma$-ray halo observations are 2-3 orders of magnitude larger than the fields produced by the cosmological version of the Biermann Battery. Possibly the Battery fields are amplified 
by strong, localized shear flows (Ryu et al. 2008), and are distributed intermittently along the line of sight. Possibly they were created by an entirely different process. Extending these pioneering observations to many more sources would put firmer constraints on the magnitude and coherence length of the field, and on its spatial distribution.

\section{Galactic Magnetic Fields}

Galactic magnetic fields are probed through several complementary techniques:

- Diffuse nonthermal continuum: Cosmic ray electrons gyrating in the interstellar magnetic field emit polarized synchrotron radiation. The orientation of the polarization depends on the orientation (but not the direction) of magnetic field projected on the plane of the sky. If the field were uniform the radiation would be about $70 \%$ polarized; lower polarization indicates unresolved or line of sight magnetic strucutre. The nonthermal continuum probes the orientation, strength, and angular dispersion of the plane of sky field, convolved with the cosmic ray electron spectrum.

- Faraday rotation: When linearly polarized radiation propagates along a magnetic field in a medium with free electrons, its plane of polarization rotates. Faraday rotation occurs in pulsars, extragalactic radio sources, and the diffuse nonthermal continuum. Faraday rotation is a probe of the strength and directedness of the line of sight field, convolved with the thermal electron spectrum.

- Zeeman splitting: Magnetic fields lift the degeneracy of magnetic sublevels in atoms and molecules. Unlike the Sun, where the full vector field is measured, only the longitudinal Zeeman effect has been observed in the interstellar medium. The longitudinal Zeeman effect effect is a probe of the strength and directedness of the line of sight field, convolved with the local density of atoms and molecules.

- Polarization by aligned dust grains: Aspherical interstellar dust grains are aligned by the interstellar magnetic field. Starlight is polarized by intervening dust, as is the thermal infrared emission from dust. Polarization probes the orientation of the plane of sky magnetic field in regions of moderate to high density.

Application of these techniques has led to a fairly consistent picture of the magnetic field within a few kiloparsecs of the Sun. The mean orientation is nearly azimuthal - the deviation is consistent with alignment along the spiral arms - and nearly parallel to the Galactic plane. The rms fieldstrength is about $5.5 \mu \mathrm{G}$, which puts the magnetic energy density near equipartition with the energy densities in random gas motion and in cosmic rays. The field has a nonzero average of about $1.6 \mu G$ over the observed volume, with the remaining field in the form of fluctuations. The azimuthal field reverses sign at least once with Galactocentric radius (Brown et al. 2007), possibly more (Han 2009). At high Galactic latitudes, the azimuthal field is antisymmetric about the Galactic plane (socalled dipole symmetry); the parity of the field near the Galactic plane is less clear. As to the fluctuation spectrum, there is evidence for an outer scale of 50 - 100 parsecs, with structure down to subparsec scales. The field declines with distance from the Galactic plane, with a scale height of about $1.5 \mathrm{kpc}$, similar to that of the diffuse ionized gas, but larger than that of the atomic and molecular gas distributions. The fieldstrength increases toward the Galactic Center.

By the nature of the magnetic field probes described above, the fieldstrength is relatively well measured in the diffuse ionized and neutral components, and in the molecular component, but not well measured in the very hot, low density component, and in the halo. Within the density range over which the field is measured, there is no relationship between fieldstrength and gas density in the diffuse ionized and neutral gas. The field does increase with gas density in the denser, molecular gas, with $B \propto \rho^{1 / 2}$ giving an 
upper envelope. However, this relationship cannot be extrapolated to the diffuse gas; the mean density of giant molecular clouds is about $50 \mathrm{~cm}^{-3}, 50$ times denser than the mean density of diffuse gas, but the mean $B$ in these clouds is only about twice that measured in the diffuse gas, not 7 times larger, as $B \propto \rho^{1 / 2}$ would suggest (Crutcher et al. 2003).

The existence of a mean vertical magnetic field, or lack of it, is a strong constraint on dynamo models. Such a field would be difficult to remove, and hence could be primordial. Mean field dynamo theories can also produce vertical fields with either dipole symmetry (constant across the Galactic midplane) or quadrupole symmetry (reversing across the midplane). Searches for a mean vertical field in the solar neighborhood have had mixed results.Taylor et al. (2009) and Mao et al. (2010) found a north-directed field of $0.30 \mu G$ below the Galactic plane. The former study reports a south directed field of $0.14 \mu G$ above the Galactic plane while the latter finds no net field there.

There is a close relationship between star formation rate, magnetic fieldstrength, and energetic particle density. The far-infrared luminosity (essentially, the luminosity of young, massive stars) and radio synchrotron luminosity are observed to be linearly proportional over a wide range of galaxy luminosities and star formation rates (Bell 2003 and references therein). Since the rate of core collapse supernovae is correlated with the star formation rate, and supernovae are the dominant source of energy in the interstellar medium, this is evidence that magnetic field amplification, cosmic ray acceleration, and supernova remnants are linked.

Galaxies, including the Milky Way, have been magnetized throughout their history. In the Milky Way this is inferred from the presence of light elements detected in the atmospheres of some of the oldest halo stars. These elements are thought to have been produced by cosmic ray spallation reactions in the material from which these stars formed (Parizot \& Drury 1999). Acceleration and confinement of cosmic rays requires a magnetic field, although it could have been several orders of magnitude weaker than the present Galactic field (Zweibel 2003). Coherent, microgauss magnetic fields have been measured in damped Ly $\alpha$ systems at redshift $z \sim 1$ (Oren \& Wolfe 1995).

\section{Stellar vs Galactic Dynamos}

The solar cycle is the best evidence for a solar dynamo. Although the roles of the convection zone, the tachocline, and the radiative core are not yet fully understood, it is clear that differential rotation, poloidal circulation, and thermal convection, together with an outer boundary condition that permits the magnetic field to escape, act jointly to produce large scale toroidal and poloidal fields which reverse over a stable 22 year period.

The evidence for a Galactic dynamo is less clear. The timescales for cyclic behavior are far too long to be detected, and the variety of magnetic field morphologies observed in other galaxies has never been interpreted as evidence for cycles. Perhaps the best argument for dynamos in galaxies is the ongoing replacement of the interstellar medium. Large disk galaxies such as the Milky Way accrete gas through mergers with other galaxies, engulfing dwarf satellites, and infall of extragalactic gas, and eject gas via winds. Gas is added to the interstellar medium through stellar mass loss, and lost through star formation. These processes result in complete replacement of the interstellar medium within $10^{9} \mathrm{yr}$, or about 4-5 rotation periods. Maintaining a coherent field of the observed strength and orientation as magnetized gas is added and deleted requires the continuous operation of dynamo processes.

Stellar and galactic dynamos operate in very different regimes, both macroscopically and microscopically. Normal stars are pressure supported, with little rotational support. 
Convective turbulence in stars is highly subsonic, and slow even with respect to rotation. Therefore, magnetic fields reach equipartition with convection and differential rotation at large plasma $\beta$. Under these conditions most of the field is in pressure confined magnetic flux ropes which occupy a small fraction of the volume. Galactic disks are rotationally supported in the radial direction, with turbulent velocities that are supersonic but well below the rotational velocity. Therefore, when the magnetic field reaches equipartition with the turbulence, it is too strong for confinement in flux ropes, but must be volume filling. Thermal and dynamical gas pressure, cosmic ray pressure, and magnetic forces jointly determine the vertical structure of galactic disks.

The mean flows in the Sun - both the differential rotation and the circulation are quite well determined from surface measurements and from helioseismology. The turbulent convection in the solar interior is not directly observed, although its primary effect, the transport of heat, is well measured. It is highly subsonic except near the photosphere, and compressibility effects over most of the solar interior are weak. The mean flow in the Galaxy is differential rotation, which is well measured. Flow perpendicular to the Galactic plane, whether a wind or a poloidal circulation or "fountain flow" driven by supernovae, has been inferred, but not directly measured. The turbulence is probably driven more by supernovae than by in situ instabilities. A combination of density and velocity diagnostics show that the turbulence exists on scales from the thermal ion gyroradius to tens of parsecs. The large scale turbulence is highly supersonic, and compressibility effects are large.

Despite these differences, some dynamical features are common to both stars and galaxies. Magnetic buoyancy plays a role in both types of system. In stars it acts radially; in galactic disks it acts vertically. Buoyancy driven escape can limit the strength of the field. The twisting of rising magnetic loops by Coriolis forces produces a net helicity flux, which may promote the growth of a large scale field (Blackman \& Field 2001). Both types of system are turbulent over a large range of scales. Neither stars nor galaxies are magnetically dominated, but magnetic fields affect the slower flows, and certainly the turbulence; therefore the dynamo is coupled to the gas dynamics.

At the microscopic level, stars and galaxies also differ substantially. The plasma in stellar interiors is highly collisional: particle collision times $\tau_{c}$ are much less than gyroperiods $2 \pi / \omega_{c}$, so transport coefficients are isotropic with respect to the magnetic field. The ratio of magnetic to viscous diffusivity, or magnetic Prandtl number $P m$, is much less than one: the kinetic energy spectrum extends to much smaller scales than the magnetic spectrum. The transport coefficients in stellar interiors are well understood at the level needed for dynamo theory: both plasma and radiation contribute to the transport, the relative amounts varying with electron degeneracy and stellar luminosity, and it is known how to calculate them. In the outer atmosphere, the physics is more complicated and less certain; partial ionization effects and kinetic effects, in particular, may affect the rate and onset conditions of magnetic reconnection, and thus magnetic flux escape.

Interstellar gas is much more complex than stellar plasma. Most interstellar gas is sufficiently collisionless $\left(\omega_{c} \tau_{c} \gg 1\right)$ that plasma transport coefficients are highly anisotropic. At the low densities typical of interstellar gas, $\mathrm{Pm} \gg 1$, so the magnetic spectrum extends far below the velocity spectrum. Interstellar gas has multiple components; most of it (by mass, but not by volume) is weakly ionized, and pervaded by cosmic rays. On large scales, the plasma and neutrals can be treated as a single, conducting fluid, but on small scales - typically less than a parsec, but much larger than the resistive scale the plasma decouples from the neutrals. At small scales, the plasma pressure and Alfvén Mach number are low $\beta \ll 1, M_{A} \ll 1$, and the medium is magnetically controlled. Partial ionization promotes the formation of current sheets (Brandenburg \& Zweibel 1994), 
which accelerates magnetic reconnection in neutral sheets (Heitsch \& Zweibel 2003). Ion neutral drift in a turbulent medium leads to fast diffusion of the magnetic field through the neutrals (Heitsch et al. 2004) and also suppresses the growth of magnetic fields at scales below the ion-neutral decoupling scale (Zweibel \& Heitsch 2008). Cosmic rays are coupled to the thermal gas by scattering from small scale magnetic fluctuations. On scales larger than their mean free path (typically a few pc), they behave as a fluid, and are a source of buoyancy. The effect of cosmic ray buoyancy on large scale dynamos was first proposed by Parker (1992). On small scales, cosmic rays amplify helical Alfvénic fluctuations, which if the cosmic ray flux is sufficently large can lead to significant magnetic field amplification (Zweibel 2003, Bell 2004). The effects of these additional components on galactic dynamos is not yet fully understood.

Again, despite their differences, stars and galaxies have common features. In both, the microscopic transport coefficients imply that the velocity and magnetic spectra extend to scales many orders of magnitude less than the global scales. This creates conceptual difficulties for the generation of large scale magnetic fields and makes direct numerical simulation of the full system all but impossible.

\section{The Ingredients of Galactic Dynamo Theory}

Certain features of the Galactic magnetic field seem easily explained. The overall strength of the field is consistent with the expectation of equipartition between turbulent kinetic and magnetic energies. Its strength and scale height are also consistent with stability arguments: if too large a fraction of the weight of the interstellar medium is magnetically supported, it is unstably stratified (Parker 1966). The predominantly azimuthal orientation of the field is naturally produced by the strong differential rotation of the Galactic disk.

None of these arguments explain the existence of the large scale field, which is coherent on scales of at least several kiloparsecs, possibly more. This scale is one to two orders of magnitude larger than the largest forcing scale in the interstellar medium: the scale of superbubbles, formed by the winds and supernova explosions of clusters of massive stars. This coherent field must be sustained over the $\sim 10^{9} \mathrm{yr}$ timescale on which it is disrupted by stellar ejecta, star formation, infall of material, and possibly a wind.

There are two current models, and an emerging third model, for the mean field. The primordial model (Howard \& Kulsrud 1997) assumes the field was present at the time the Galactic disk formed, and inclined at an angle to it. The vertical magnetic flux associated with this field is almost impossible to remove, and is a source of azimuthal field through windup by differential rotation. In the absence of diffusion, the windup amplifies the field linearly with time and produces reversals with radius on a scale $\Delta r \sim R / N$, where $R$ is the radius of the disk and $N$ is the number of times the disk has rotated. The windup is counteracted by diffusion, which HK take to be ion-neutral drift perpendicular to the Galactic plane. One difficulty with the model is the presence of the thick ionized gas layer, which envelopes the neutral hydrogen disk and blocks ion-neutral drift. The second difficulty is that no vertical field which runs through the Galactic plane has yet been detected, and it is even possible that there is a vertical field which reverses across the Galactic plane, contrary to the model. However, the primordial model is appealing in its simplicity, and some aspects of the scenario it describes may be relevant to galaxies.

The second model is the mean field dynamo, which was first applied to the Galaxy by Parker 1971. Ferrière (Ferrière 1993a, Ferrière 1993b) has calculated the $\alpha$ and $\beta$ tensors when the small scale induction and diffusion are produced by expanding shells associated with supernova remnants and superbubbles. Hanasz \& Lesch 1993 and Kowal et al. (2006) 
calculated $\alpha$ for Parker instabilities driven by cosmic ray buoyancy. Calculations of mean field dynamo modes, some incorporating presecriptions for nonlinear saturation, have been calculated, for example, by Ferrière \& Schmitt (2000). Unstable modes with both dipole and quadrupole symmetries are, with growth times typically in the $10^{8}-10^{9}$ year range. These models make clear predictions, and have been used to interpret observations. However, they encounter the objections that plague mean field dynamo theory in any system with large magnetic Reynolds number $R m$ : the small scale fields are predicted to grow much faster than the mean field (Kulsrud \& Anderson 1992. The existence of dominant magnetic power on the resistive scale - about $R_{\odot}$ - is firmly precluded by observations.

A third type of model is direct numerical simulation of the gas dynamics in galaxies (e.g. Kulesza-Zydik et al. 2010). Such work can include a variety of interstellar processes, and make no assumptions about mean field theory. Like mean field models, direct numerical simulations can be compared to observations of galaxies. However, they are of necessity restricted to large scales, and therefore do not address the problem of dominance of the small scales.

Although we lack a complete explanation for the properties of the Galactic magnetic field, we can make some educated guesses as to what the ingredients of such a theory would be:

- Seed magnetic field: As discussed in $\S$, possible sources include a "primordial" field generated by an exotic physical process in the early Universe, a large scale intergalactic field generated by the Biermann Battery operating in shock and/or ionization fronts, or a field from stellar ejecta magnetized by battery and dynamo processes. The resulting magnetic field is not completely independent of initial conditions, as the net vertical magnetic flux through the galaxy should be preserved. Since coherent $\mu \mathrm{G}$ magnetic fields seem to have existed by the time the Universe was $1 / 10$ its present age, the seed field must be large enough to grow to the observed level in $10^{9} \mathrm{yr}$. For example, if $B=B_{i} e^{t / \tau}$ and $\tau=10^{8} \mathrm{yr}$, we must have $B_{i} \geqslant 5 \times 10^{-11} \mathrm{G}$. Magnetic field estimates from stellar ejecta satisfy this constraint, fields produced in cosmological fronts do not, unless they are pre-amplified by flows in the intergalactic medium. Even very weak magnetic fields can be amplified by plasma mechanisms. For example, Krolik \& Zweibel (2006) showed that accretion disks are unstable to magnetorotational instabilities even when the ions are unmagnetized. However, the wavelength of the fastest growing modes scale inversely with $B$. In collisional disks, the modes are therefore subject to strong damping, but in collisionless disks they can survive. Thus, the battery to MRI scenario appears to work, at least in hot, low density disks. Other amplification mechanisms may exist in cold, dense disks, such as protoplanetary disks (Tan \& Blackman 2004). Once the battery fields are amplified, the next step is to eject them, in supernova explosions, jets, or winds. Then they may remain in the ambient medium as confined structures, or they may diffuse throughout the volume. This seems to be a plausible mechanism for magnetizing the interstellar medium. For example, if we assume a turbulent lengthscale of $10 \mathrm{pc}$ and a characteristic velocity of $10 \mathrm{~km} \mathrm{~s}^{-1}$, the turbulent diffusivity $D_{t}$ of the interstellar medium is $D_{t} \sim 3 \times 10^{25} \mathrm{~cm}^{2} \mathrm{~s}^{-1}$, the rms displacement of magnetized material is $300 \mathrm{pc}$ in $10^{9} \mathrm{yr}$. Empirically, the apparently uniform chemical composition of interstellar gas in the solar neighborhood is good evidence that the interstellar medium is well mixed.

- Energy source: Galactic gas dynamics are determined by gravity, which is due primarily to dark matter and stars, and by energy from massive stars, in the form of radiation, winds, and supernova explosions. The correlation between far-IR luminosity and synchrotron luminosity is evidence that energy input from massive stars is important in 
driving the dynamo. Stellar energy drives turbulence, while cosmic ray acceleration at shock fronts amplifies small scale magnetic fields.

- Fieldline stretching: Fieldine stretching is fundamental to any dynamo. In galactic dynamos, fieldlines are stretched by differential rotation, small scale turbulence, expansion of bubbles, and possibly a galactic wind or fountain flow. Accretion of intergalactic gas clouds or dwarf galaxies, and mergers with larger galaxies, generate large tidal forces, which may help to drive a dynamo. and by tidal stretching in mergers. Turbulence seems to exist over a large range in scales, and because $\mathrm{Pm} \gg 1$, the fieldlines develop structure on scales far below the viscous cutoff for the velocity scale.

- Fast diffusive transport: Stellar ejecta is undermagnetized in comparison with the interstellar medium; infalling intergalactic material may also be undermagnetized. Magnetic fields must diffuse rapidly into this material, possibly by ion - neutral drift, acting jointly with turbulence, or by fast magnetic reconnection. In models with buoyancy driven escape, the field must separate itself from the matter, by reconnection or diffusion. The problem of fast diffusive magnetic field transport is related to the problem of thermal and chemical mixing of interstellar gas.

- Fast magnetic reconnection: Dynamos require magnetic reconnection to irreversibly change magnetic topology. Since reconnection typically operates at small scales, it can also suppress the growth of small scale fields. Classical reconnection rates in the interstellar medium are very low, but a number of mechanisms have been proposed to accelerate the reconnection rate (Zweibel \& Yamada 2009). Among these are current sheet formation by ion-neutral drift (Brandenburg \& Zweibel 1994), externally driven MHD turbulence in the reconnection layer (Lazarian \& Vishniac 1999), and self-disruption of the reconnection layer by the plasmoid instability (Loureiro et al. 2007), possibly accompanied by kinetic effects. Rapid progress is being made in this area.

\section{Summary}

This paper is a short review of the galactic dynamo problem, with comparison to the stellar dynamo problem. The best evidence that dynamos operate in galaxies is the ongoing replacement of their interstellar gas. The greatest challenge in galactic dynamo theory is explaining the existence of a coherent magnetic field, comparable in magnitude to the random field, over scales much larger than the forcing scale, Such fields have existed at least since the Universe was about 1/10 its present age.

The seed magnetic fields for the dynamo early in the history of the Galaxy are more likely to have been produced by many small sources than by a cosmological scale Biermann Battery process. The fields produced by the latter cannot be amplified to $\mu G$ levels in $1 / 10$ the age of the Universe. Intriguing recent evidence for an intergalactic field 2-3 orders of magnitude stronger than predicted by the Battery mechanism may hint at a true intergalactic dynamo, or at a magnetogenesis mechanism based on exotic physics.

Galactic dynamos differ from stellar dynamos. The interstellar medium is relatively collisionless, much of its mass is electrically neutral, it is pervaded by cosmic rays, and its turbulence is supersonic. Unlike stars, which accommodate many magnetic cycles over their lifetimes, galactic gas is replaced in 4-5 rotation periods, and galaxies themselves are no older than $\sim 50$ rotation periods.

Galactic dynamo theory needs a breakthrough. It may come through basic plasma physics, such as improved understanding of the small scale dynamo in a medium with anisotropic viscosity, or of magnetic reconnection under interstellar conditions. It may come as astrophysical processes such as gas dynamics during galaxy mergers and impacts of intergalactic or galactic halo clouds on galaxy disks are more accurately modeled and 
better understood. New observations may modify our characterization of the galactic dynamo problem, and the basic constraints. Each of these aspects is fascinating on its own.

I met Juri Toomre at a solar physics meeting in 1978. He was as friendly, inquisitive, canny, witty, and bald then as he is now, in 2010. We were colleagues from 1981, when I became a faculty member at the University of Colorado, until 2002, when I left for the University of Wisconsin. I learned an enormous amount from him about science itself, and about being a scientist. He created many opportunities for me and was very helpful to me in my own career and in being effective as both Department Chair and JILA Chair. He probably helped me also in ways that I will never know. This meeting has been a wonderful way to witness Juri's enormous impact on the lives of other many other scientists, and on science.

I am happy to acknowledge the support of the National Science Foundation through grants PHY0821899 and AST0907837.

\section{References}

Ando, S. \& Kusenko, A. 2010 ApJ, 722, L39

Beck, R. 2009 Ast. 83 Sp. Sci. Trans, 5, 43

Bell, E. F. 2003 ApJ, 586, 794

Bell, A. R. 2004 MNRAS, 353, 550

Blackman, E. G. \& Field, G. B 2001 Phys. Plasmas, 8, 2407

Brandenburg, A. \& Zweibel, E. G. 1994 ApJ 427, L91

Brown, J. C., Haverkorn, M., Gaensler, B. M., Taylor, A. R., Bizunok, N. M., Dickey, J. M., \& Green, A. J. 2007 ApJ, 663, 258

Crutcher, R. M., Heiles, C., \& Troland, T 2003 Turbulence $\&$ Magnetic Fields in Astrophysics, eds. E. Falgarone 85 T. Passot, Lecture Notes in Physics, 614, 155

Ferrière, K. M. 1993 ApJ, 404, 162

Ferrìre, K. M. 1993 ApJ, 409, 248

Ferrière, K. M. \& Schmitt, D. $2000 A \& A 358,125$

Furlanetto, S. R. \& Loeb, A. 2001 ApJ, 556, 619

Gnedin, N. Y., Ferrara, A., \& Zweibel, E. G. 2000 ApJ, 505, 516

Han, J. L. 2009 Cosmic Magnetic Fields: Proceedings of IAU Symposium 259, 455

Hanasz, M. \& Lesch, H. 1993 AEAA, 278, 561

Heitsch, F. \& Zweibel, E. G. 2003 ApJ 583, 229

Heitsch, F., Zweibel, E. G., Slyz, A., \& Devriendt, J. E. G. 2004 ApJ, 603, 175

Howard, A. M. \& Kulsrud, R. M. 1997 ApJ, 483, 648

Kowal, G., Otmianowska-Mazur, K., \& Hanasz, M. 2006 A\&SA, 445, 915

Krolik, J.H. \& Zweibel, E. G. 2006 ApJ, 644, 651

Kronberg, P. P., Dufton, Q. W., Li, H., \& Colgate, S. A 2001 ApJ, 560, 178

Kulesza-Zydik,B., Kulpa-Dybel, K., Otmianowska-Mazur, K., Soida, M., \& Urbanik, M. 2010 $A \mathscr{E A}, 522,61$

Kulsrud, R. M. \& Anderson, S. W. 1992 ApJ, 396, 606

Kulsrud, R. M., Cen R., Ostriker, J. P., \& Ryu, D. 1997 ApJ, 480, 481

Kulsrud, R. M. \& Zweibel, E. G. 2008 Rep. Prog. Phys, 71, 046901

Lazarian, A. \& Vishniac, E. T. 1999 ApJ, 517, 700

Loureiro, N. F., Schekochihin, A., \& Cowley, S. C 2007 Phys. Plasmas, 14, 100703

Mao, S. A., Gaensler, B. M., Haverkorn, M., Zweibel, E. G., Madsen, G. J., McClure-Griffiths, N. M., Shukurov, A., \& Kronberg, P. P. 2010 ApJ, 714, 1170

Neronov, A. \& Vovk, I 2010 Science, 328, 73

Oren, A. L. \& Wolfe, A. M. 1995 ApJ, 425, 624

Parizot, E. \& Drury, L. $1999 A \& A, 349,673$

Parker, E. N. 1966 ApJ, 145, 811 
Parker, E. N. $1971 A p J, 166,295$

Parker, E. N. 1992 ApJ, 401, 137

Rees, M. J. 1987 QJRAS, 28, 197

Ryu, D., Kang, H., Cho, J., \& Das, S. 2008 Science, 320, 909

Tan, J. C. \& Blackman, E. G. 2004 ApJ, 603, 401

Taylor, A. R., Stil, J. M., Sunstrum, C. 2009 ApJ, 702, 1230

Widrow, L. M. 2002 Rev. Mod. Phys., 74, 775

Zweibel, E. G. 2003 ApJ, 587, 625

Zweibel, E. G. \& Heitsch, F. 2008 ApJ, 684, 373

Zweibel, E. G. \& Yamada, M 2009 ARAA 47, 291

\section{Discussion}

Steve ToBias: There are some theories that have the kinematic field generated on the resistive scale followed by a scale by scale saturation. Is this feasible given the timescale?

ELLEN ZWEIBEL: The turnover times of the largest eddies are probably of order $10^{6} \mathrm{yr}$, which is quite short compared to the replacement time for the interstellar medium. The field is observed to be coherent on scales 10 - 30 times larger than the largest eddies. I'm not sure how to estimate the time required for amplification on the global scale.

Axel BrandenBuRg: The growth rate of the MRI may not be relevant because the MRI relies on the existence of an existing background field, which still needs to be amplified by a dynamo. I would guess that this mechanism is only when there are no other sources of turbulence, e.g. in the outskirts of the galaxy? In any case I do not think this MRI dynamo would be more efficient than the turbulent dynamo. Also, it would only produce large scale fields if the usual ingredients (stratification, rotation) are present.

ELLEN ZwEIBEL: Krolik and I contend that the Battery fields in accretion disks become unstable to the MRI when they are still very weak. To the extent that the MRI acts as a dynamo, a large scale field will then be amplified. Of course, there are other possible sources of turbulence, e.g. gravitational instabilities in protostellar disks, where the MRI is likely to be damped by collisional processes.

AnNick Pouquet: Is equipartition between rms magnetic field and turbulent gas pressure exact, or is there a winner?

ELLEN ZWEIBEL: The observations refer only to averages, so I don't think it's possible to to say.

Keith Moffatт: Turbulence in the interstellar medium is characterized by very large magnetic Prandtl number. Can you estimate the Kolmogorov scale of the turbulence on which the most efficient stretching and intensification of the magnetic field takes place? I discussed this problem as early as 1962:JFM (magnetic eddies...)

ELLEN ZwEIBEL: If one uses the parallel viscosity coefficient and assumes a fully ionized gas with $T=10^{4} \mathrm{~K}, n=1$, and an outer scale of 30 parsec, then the Kolmogorov scale is about $10^{14} \mathrm{~cm}$, or $7 \mathrm{AU}$ - very small. 